\title{
Damage to the Vascular Endothelium as a Leading Mechanism of COVID-19 Systemic Pathology
}

\author{
O. A. Gomazkov* \\ Orekhovich Scientific Research Institute of Biomedical Chemistry, Moscow, Russia \\ *e-mail: oleg-gomazkov@yandex.ru \\ Received November 17, 2020; revised November 17, 2020; accepted November 17, 2020
}

\begin{abstract}
An analysis of the molecular and pathophysiological mechanisms of COVID-19 is presented. The endothelium of blood vessels, a kind of "endocrine tree" of the lungs and other organs in which important pathophysiological processes are concentrated, is considered as the target of the aggressive effects of the SARS-CoV-2 coronavirus. Since the main cellular target of viral aggression is the ACE2 enzyme, consideration of its role is the main line of discussion. Coronavirus blocks the activity of ACE2, a natural producer of angiotensins peptides, thus disrupting the balance of hemovascular control. Under normal conditions this mission is performed by the ACE/ACE2 complex, enzymes that control the synthesis and physiological activity of angiotensins and bradykinin peptides. Changes in the ACE/ACE2 axis ratios and cytokine stress are associated with endothelial dysfunction and a number of vascular disorders. The STORM-2 concept is proposed for the first time. According to the concept, the cause of severe organ pathology is the violation of hemostasis, transcellular diffusion, and maintenance of blood pressure.
\end{abstract}

Keywords: COVID-19 pandemic, cytokine stress, vascular endothelium, angiotensin-converting enzyme-2, angiotensin-bradykinin axis, STORM-2, organ damage

DOI: $10.1134 /$ S2079086421060049

\section{INTRODUCTION}

Due to the extremely rapid development of events associated with the COVID-19 pandemic, an important task is to analyze the pathophysiological and molecular aspects of the disease, which cause a complex set of negative processes.

The outbreak of this infection was caused by a variant of the coronavirus of the SARS-CoV group. The World Health Organization officially named the COVID-19 infection (Coronavirus disease 2019). The International Virus Taxonomy Committee officially named the new pathogen SARS-CoV-2.

The most characteristic manifestation of COVID-19 pathogenesis is acute lung injury with an inverted response of the immune systems. The "cytokine storm," which is initially caused by a viral attack, demonstrates a clinical picture of proinflammatory etiology with manifestations of hemodynamic instability, dysfunction of many body organs, and neurological dissonance. Systemic vascular lesions, primarily of the lungs but also of the heart, brain, kidneys, endocrine organs, intestines, etc., are pronounced in various forms of this disease.

The main cellular target of viral aggression is the second type of angiotensin-converting enzyme (ACE2, angiotensin-converting enzyme 2), a natural biochemical factor of vascular regulation. Due to its very high affinity, the SARS-CoV-2 coronavirus blocks the activity of ACE2, disrupting the balance of cytoimmune and hemovascular control. Under normal conditions, this mission is carried out by a complex of related enzymes, ACE and ACE2, which control the synthesis and physiological activity of bradykinin and angiotensins. Specific acceptance of the ACE2 enzyme by the coronavirus and, as a consequence, the prevalence of its antipode ACE reveals dissonance in regulation, with dysfunction of endothelial cells and multiple disorders in the hemostatic system.

The second aspect of the problem relates to the role of the endothelium, the monolayer of cells lining the vascular surface. Since the vascular endothelium is the main site of localization of the enzymes ACE and ACE2 in the body, damage to the cells of the lungs and other organs is a typical picture of the systemic pathology of COVID-19. Endothelial disruption turns out to be a key process in the "disorder" of hemostasis control, vascular tone, transcellular diffusion, microcirculation, and blood-pressure maintenance. The imbalance of the ACE/ACE2 biochemical axis due to enhancement of the destructive activity of angiotensin's peptides leads to prolongation of the effects of cytoinflammation, thrombogenesis, and prohypertensive activity. 
Thus, based on the above, the COVID-19 clinical materials, and data from publications of the last period, the term is proposed STORM-2. Its essence relates to the manifestation of multiple disorders in which cellular inflammation is accompanied by microthrombosis and hemorrhage with symptoms of intravascular blood coagulation and multiple organ dysfunction. This negative picture is characteristic not only of lung damage, in which the impact of coronavirus is primary. In the acute course of the disease, the recorded violations of the functions of the heart, brain, kidneys, endocrine system, etc., were expressed in the form of coagulopathy and microthrombosis dissemination.

The concept of STORM-2 involves the ability to apply a special pharmacotherapy strategy for COVID-19, the key component of which is normalization of the endothelium and management of the blood coagulation system, transcellular diffusion, and maintenance of blood pressure with an effect on the corresponding pharmacological targets.

\section{PANDEMIC COVID-19: DISSONANCE OF PATHOCHEMICAL PROCESSES}

The complexity and variety of manifestations of coronavirus disease is indicative of a complex of dissonances of pathochemical processes in the body.

Clinical studies have found that COVID-19 can be asymptomatic or have moderate or severe manifestations, e.g. pneumonia, respiratory failure, organ coagulopathy, and neurological disorders. The results of radiographic and computed tomography of the lungs detected multiple focal changes in the vessels already in the early stages in the severe course of the disease.

The initial stage of infection is the penetration by the SARS-CoV-2 coronavirus of cells containing the membrane-bound protein ACE2. The primary, quickly attainable target is the alveolar cells of the lungs, which leads to the development of pneumonia. Immunological complications such as macrophage activation, which leads to cytokine storm syndrome, and acute respiratory distress syndrome are typical of COVID-19 patients. Inflammation-inducing cytokines, including tumor necrosis factor (TNF), interferon- $\gamma$, interleukins IL-1, IL-6, IL-18, etc., are secreted in large amounts.

The most common clinical manifestation of COVID-19 is bilateral pneumonia, a diffuse alveolar injury with severe microangiopathy. Hypercoagulable syndrome develops in some patients, and other organs and systems are also affected: heart, brain, kidneys, liver, gastrointestinal tract, and the endocrine and immune systems. Patients with severe COVID-19 develop vascular endothelial dysfunction accompanied by intracellular diffusion, coagulopathy, and thrombosis (Tang et al., 2020).
Thus, the clinical development of COVID-19 includes several phases:

(a) viral infection and a complex of hyperimmune, proinflammatory responses in the form of a cytokine storm;

(b) violation of the vascular endothelium of the pulmonary, coronary, cerebral, and other systems;

(c) coagulation and thrombotic complications in the organ and disseminated forms;

(d) neurological disorders of a direct and distant nature.

In line with this, the therapy for various forms of COVID-19 uses appropriate means to contain dissonance and maintain tactics of protective treatment.

\section{ANGIOTENSIN-CONVERSING ENZYMES AS A SYSTEM OF FUNCTIONAL PROTEINS INVOLVED IN PATHOGENESIS}

Angiotensin-converting enzyme (ACE) is traditionally considered the leading factor in the reninangiotensin system, which is associated with the control of heart and vascular diseases. The Institute of Biological and Medicinal Chemistry of the Russian Academy of Medical Sciences in Moscow was involved in the discovery of this enzyme, which was originally called carboxycatepsin. In the laboratory of Academician V.N. Orekhovich, among a number of proteolytic proteins, a catalytic unit with the properties of a dipeptidylcarboxypeptidase was first isolated and introduced into the world nomenclature of enzymes as EC 3.4.15.1 (Eliseeva and Orekhovich, 1963). Along with this, this list includes a study by Skegs et al. (1956) of a hypertensin-converting enzyme, which has gained great popularity as one of the most important regulators in cardiology.

The role of angiotensin convertase as a specific peptide hydrolase is systemic, since it was found that it simultaneously participates in the formation of the prohypertensive peptide angiotensin II (ANG-1-8) and in the hydrolysis of its physiological antipode, bradykinin, a product of the kallikrein-kinin system (Vane, 1969). It is essential for the subsequent presentation to note that immunochemical studies identified the prevailing localization of ACE on the luminal surface of lung endothelial cells (Ryan et al., 1975). The medical aspect of ACE research was confirmed by the discovery of conjugated factors of regulation of the blood and the vascular system (nitric oxide, prostaglandins, endothelin, prostacyclins, etc.), which now constitute a complex system of tissue biochemical processes in health and in various diseases.

Consideration of ACE as kininase II is impossible without its functional "enemy," the enzyme kallikrein, a representative of the kallikrein-kinin system that is also involved in blood pressure control. Subsequent work made it possible to talk about an integral axis of biochemical components involved in many normal 
and pathological processes in the body: ACE $\rightarrow$ angiotensin II $\rightarrow$ receptors $\rightarrow$ physiological effects and, as a counterbalance: Hageman factor $\rightarrow$ kallikrein $\rightarrow$ kininogen $\rightarrow$ bradykinin. It should first be noted the relationship of these systems with the endothelium of the lungs in the control of hemovascular equilibrium (Gomazkov and Komissarova, 1976).

In 2000, type-2 angiotensin converting enzyme (ACE2) was discovered (Donoghue et al., 2000; Tipnis et al., 2000). It was found that the catalytic domains ACE2 and ACE are similar and are involved in the processing of various physiologically significant fragments of the large angiotensinogen polypeptide or in the hydrolysis of bradykinin. However, unlike ACE, ACE2 cannot hydrolyze bradykinin or neurotensin. It is also essential that ACE2 is not inhibited by lisinopril or captopril.

In the idea of a new form, ACE2, a prophetic question arose: what is new in the renin-angiotensin system? (Warner et al., 2004). History does take a dramatic turn: current research is establishing a role for ACE2 as a major cause of severe acute respiratory syndrome with SARS-CoV-2.

The fundamental position determining the specificity and scale of the COVID-19 infection is the coincidence of chemical structures that give SARS-CoV-2 coronavirus an exceptional ability to bind to ACE2 in host cells. The level of SARS-CoV-2 binding to ACE2 is 10-20 times higher than the affinity for ACE2 of the previous viral strain, SARS-CoV. On these grounds, ACE2 was presented as a functional receptor SARSCoV-2 (Li et al., 2003). Due to its special spine structure, the new virus uses ACE2 as a "Trojan horse" to enter the host cell. This circumstance, on the one hand, identifies the initial stages of a complex disease, and, on the other hand, determines the target orientation of the development of specific proteins (vaccines) as virus blockers (Tai et al., 2020).

ACE2 is affected in many human organs, but alveolar epithelial cells of the lungs are the primary target of SARS-CoV-2 (Zou et al., 2020). Pathochemical analysis also established the localization of ACE2 in humans in the endothelium of arterial and venous vessels and in the arteries of smooth muscles of almost all organs. ACE2 molecules associated with viral "reception" were found in the mucous membranes of the nose, mouth, stomach, intestines, etc. in the first stage of viral invasion (Hamming et al., 2004). This information on the multiple organ spread of ACE2 indicates the possibility of a large spread of infection and various manifestations of the pathology of COVID- 19 .

\section{ACE/ACE2 MOLECULAR AXIS AND CYTOKINE-STORM MECHANISM}

The attachment of the SARS-CoV-2 glycoprotein spike to ACE2 triggers processes that lead to hyperinflammation. Several molecular lines with overproduc- tion of inflammatory cytokines and the initiation of a cytokine storm have been considered.

Along with data from immunochemical analysis, the idea associated with the role of ACE2 itself, the main "target" of coronavirus aggression, turns out to be significant. The special role of ACE and ACE2 is their ability to influence the formation of various fragments of angiotensin I (ANG1-10). The initially functional axis, ACE2 $\rightarrow$ ANG(1-7) $\rightarrow$ MasR receptor, is in opposition to the axis ACE $\rightarrow$ ANG(1-8) $\rightarrow$ AT1 R receptor. Coronavirus suppression of the ACE2 receptor leads to a violation of the ratio inducing the expression of proinflammatory factors (Fig. 1).

New data indicate the special role of ANG(1-7) and bradykinin as initiators of proinflammatory reactions (Mahmudpour et al., 2020). An important protective function of membrane-bound ACE2 is the formation of ANG(1-7). Initially, ANG(1-7) has antiinflammatory activity due to the expression of signal transducer and transcription proteins NF- $\kappa \mathrm{B}$, MAPK, and ERK 1/2. The effects of ANG(1-7) are realized via interaction with MasR receptors in the lung epithelium and smooth muscles of the bronchi, counteracting the development of inflammatory processes (Souza Santos et al., 2018).

However, suppression of the ACE2 enzyme by coronavirus may be accompanied by a leveling of these functions. A decrease in the formation of the ANG(1-7) peptide leads to cytokine stimulation and the development of inflammatory responses (Gheblawi et al., 2020).

The history of confrontations continues when one considers components of the kallikrein-kinin system. The dissonance caused from ACE2 blockage by coronavirus brings to the fore an increase in the expression of ACE, in this case, kininase II. As a result, there is a decrease in the positive cellular effects of bradykinin and, conversely, an increase in the level of [des-Arg9] bradykinin, which is known to be a proinflammatory agent of the pulmonary epithelium. The result of kinin cytotoxicity is massive neutrophil activity and an increase in the negative phenomena associated with the severe form COVID-19 (van de Veerdonk et al., 2020). The kinin receptor BKB1R, which is activated by cytokines such as IL-1B and TNF- $\alpha$, appears to be a component of the proinflammatory cellular process. There is evidence that the use of a receptor blocker can be used to treat acute respiratory distress syndrome (Barth et al., 2012; Qadri and Bader, 2018).

\section{VASCULAR ENDOTHELIUM AS A TARGET OF SYSTEMIC DAMAGE \\ Endothelium as an Endocrine Tree, $a$ "Maestro of Blood Circulation"}

The concept of the metabolic or biotransforming function of the lungs, in which active synthesis or degradation of the most important biochemical sub- 


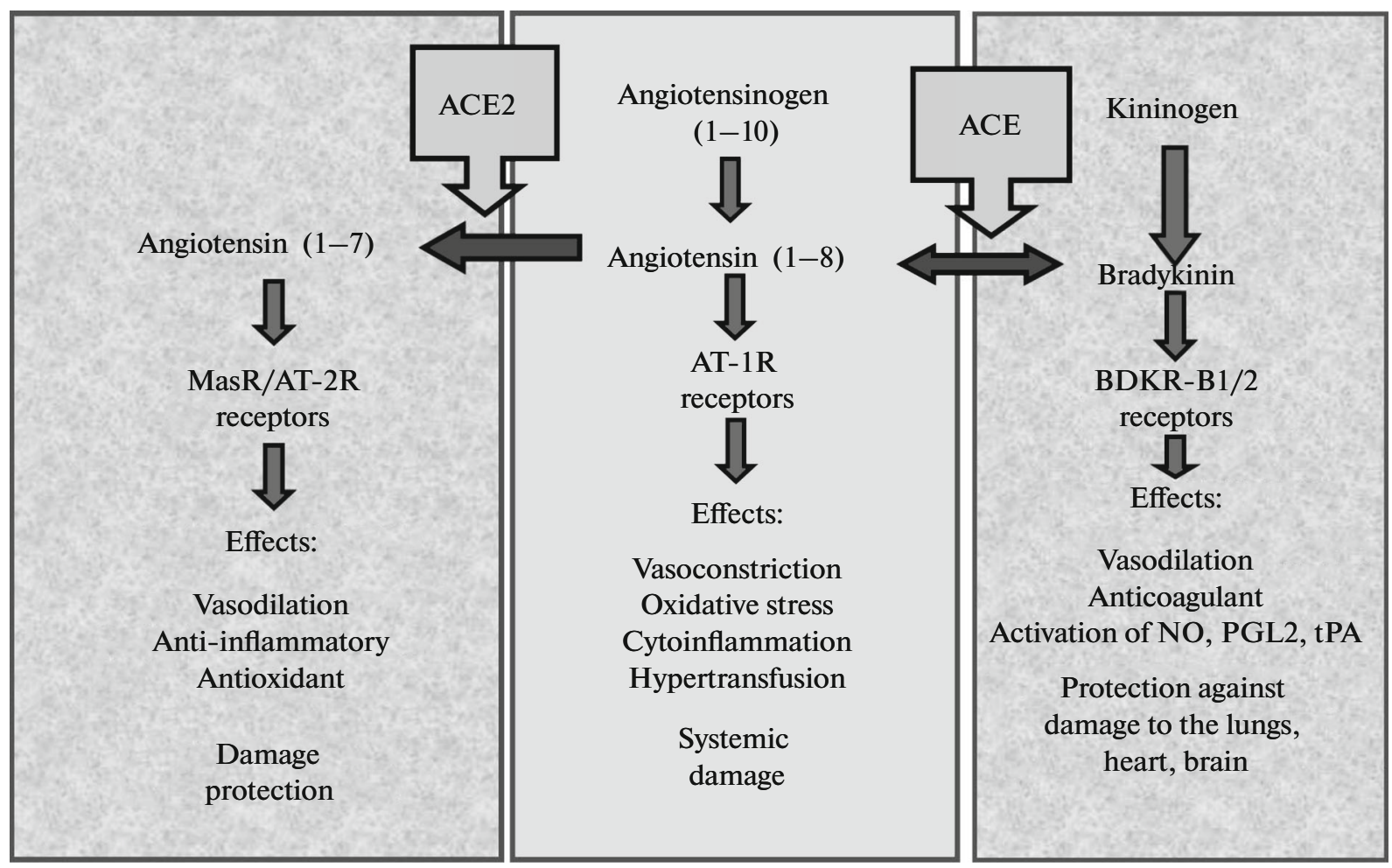

Fig. 1. Ratio of the biochemical axes of ACE and ACE2 and the imbalance in ANG(1-7), ANG(1-8), and bradykinin. Functional opposition and interactions of peptides with receptors determine the protective or pathogenetic effects. The aggressive interaction of the coronavirus with the ACE2 enzyme levels its protective mission, reduces the activity of bradykinin due to ACE, and stimulates the hyperproduction of $\mathrm{ANG}(1-8)$, causing multiple violations of vascular homeostasis.

stances occurs during the transition from venous to arterial blood, was popular in the 1970s-1980s. According to the materials of that time, biotransformation humoral mediators are exposed: adrenaline, nitric oxide, angiotensin I and II polypeptides, bradykinin, endothelin, prostaglandins E1 and E2, cyclic nucleotides cAMP and cGMP, prostacyclins, etc. The lungs play the role of a functional filter that determines the quantitative, qualitative, and, in fact, regulatory composition of biochemical components of the entire hemodynamic system (Dubiley et al., 1987).

The second aspect of this concept related to the allocation of the role of the endothelium as the "endocrine tree," or a "maestro of blood circulation," according to the characteristics of the Nobel laureate J. Vane (2001). According to current views, the endothelium not only regulates endothelium-dependent vasodilation but also actively suppresses thrombosis, vascular inflammation, and hypertrophy. A healthy endothelium exhibits a vasodilatory phenotype due to the high activity of the relaxation factors nitric oxide (NO) and prostacyclin (PGI2) and low levels of reactive oxygen species (ROS) and uric acid. The anticoagulant activity includes low levels of plasminogen activator inhibitor-1 (PAI-1), von Willebrand coagulation factor (vWF), and P-selectin. As regulators of cellular inflammation, endothelial cells secrete vascu- lar cell adhesion molecules (sVCAM), intercellular adhesion molecules (sICAM), E-selectin, C-reactive protein, TNF- $\alpha$, and IL-6.

In response to the expression of proinflammatory cytokines, endothelial cells secrete factors TNF- $\alpha$ and PAF upon endothelial dysfunction. They stimulate platelet aggregation and neutrophil adhesion. Numerous proinflammatory products are also activated, including cytokines (IL-1, TNF- $\alpha$ ), chemokines (IL-8, MCP-1), and adhesion molecules (ICAM-1, VCAM-1, E-selectin), which serve as signals for recruitment leukocytes to areas of damage. Endothelial disorders affecting hemodynamics as a whole or individual organs are the cause of many diseases (Gomazkov, 2000; Rajendran et al., 2013).

Endothelium-coupled regulators of thrombogenesis and the vascular wall tone are considered in the third aspect of the concept. Since the angiotensinconverting enzyme (ACE) finds its "home" in the endothelial caveolae, the renin-angiotensin (angiotensins I and II) and kallikrein-kinin (bradykinin, "Hagemann's factor system") ligament of components of the hemovascular homeostasis ratio was described by the vascular-wall tone, and the effect of enzyme inhibitors (Gomazkov and Trapeznikova, 1978; Kalinina et al., 1997). 


\section{Endothelial Dysfunction as Continuation of the Cytokine Storm}

The symptoms that manifest with COVID-19 infection, e.g., high arterial pressure, thromboembolism, kidney disease, neurological disorders, and diabetes, all indicate that the virus is targeting the endothelium, one of the most important tissue systems in the body. An article by Sardou et al. on the analysis of multiple organ abnormalities poses a key question: "Is Covid-19 an endothelial disease?" (Sardu et al., 2020). It can be taken as a starting position that the SARS$\mathrm{CoV}-2$ virus attack induces the hyperactivation of cytokines and chemokines. A certain sequence of immune defense is noted; however, it takes the form of stochastic disturbance, in which the protective mission is combined with overexpression and the destruction of the liquidators themselves (ShimabukuroVornhagen et al., 2018; Ye et al., 2020).

A consequence of the rapid replication of the virus and the proinflammatory response of cytokines is the stimulation of apoptosis with damage to microvessels and cell barriers with the development of alveolar edema and pulmonary and systemic hypoxia. Thus, chains of immune responses that induce each other, disrupting the sequence and adequacy of processes, play a primary role in the pathogenesis of acute respiratory syndrome COVID-19 (Merad and Martin, 2020; Mcgonagle et al., 2020).

Pathogenesis enters a critical phase upon the development of disorders associated with disorders of the vascular endothelium. Endothelial activation occurs initially within the microcirculation, including microthrombi and capillary hemorrhages. Clinical evidence suggests that cytokine-induced dysfunction, especially in the second stage of the disease, has a multiorgan continuation: arterial hypertension, myocardial damage, diabetes, and neurological complications that correspond to the principle of generalized disorder (Pearce et al., 2020). Disruption of the antithrombotic endothelial surface, along with weakening of fibrinolysis, affects the development of general and disseminated thrombogenesis (Marchandot et al., 2020). Clinical markers, such as D-dimer, a product of fibrin degradation, predict a negative nature of the pathology, reflecting the dissonance of biochemical cascades and the stimulation of coagulopathy.

\section{FAMILY OF ANGIOTENSINS AND BRADIKININ. IMPAIRMENT OF EQUILIBRIUM AS A CAUSE OF POLYORGAN PATHOLOGY COVID-19}

For the pathobiochemical mechanisms of COVID-19, special attention is drawn to the dissonance of the biochemical axes of the regulation of hemovascular homeostasis. Under normal conditions, equilibrium (more precisely, functional homeokinesis) is carried out in the framework of opposition ACE $\rightarrow$ ANG(1-8) $\rightarrow$
AT1R receptor//ACE2 $\rightarrow$ ANG(1-7) $\rightarrow$ MasR receptor. It is generally accepted that $\mathrm{ANG}(1-8)$, a product of ACE hydrolysis, is one of the main factors causing endothelial dysfunction. This is confirmed by a complex of reactions that lead to damage: oxidative stress, proinflammatory and prothrombotic activity, vasoconstriction, and changes in vascular permeability (Williams et al., 1995; Victorino et al., 2002). Longterm effects are also characteristic of ANG(1-8), including myocardial hypertrophy, vascular remodeling, and renal fibrosis.

Unlike the "first" enzyme, ACE-axis, the biochemical axis ACE2 $\rightarrow$ ANG(1-7) $\rightarrow$ Mas1R serves as protection in the form of anti-inflammatory and antithrombotic mechanisms. The positive role of ANG(1-7) is realized by the Mas-receptors of the platelets, the activation of which increases NO production and enhances the antiaggregatory properties. Targeting vascular endothelial receptors and blood factors, in opposition to the angiotensin and kinin systems, serves to maintain the hemovascular balance and to reduce the risk of vascular excesses (Fang and Schmaier, 2020).

Aggressive suppression of ACE2 coronavirus changes the picture of the ACE/ACE2 opposition towards a prevalence of the effects of $A N G(1-8)$ and the potentiation of pathogenesis. Thus, the intrigue associated with COVID-19 is to level the role of ACE2 and to increase the negative activity of ACE products. Thus, it is significant that the level of ANG(1-8), a product "liberated" with ACE, was markedly increased in the blood of patients with COVID-19 and is linearly related to the degree of lung damage (Liu et al., 2020). This finding has implications for an understanding of the strategy of the use of COVID-19 treatments. Modern pharmacology has a complex of substances blocking the corresponding receptors for bradykinin and angiotensins.

Verdecchia et al. (2020) believe that ACE2 suppression due to viral infection may be especially unfavorable in patients with a baseline (age-related) ACE2 deficiency; it increases dysregulation between the negative axis $\mathrm{ACE} \rightarrow \mathrm{ANG}(1-8) \rightarrow \mathrm{AT} 1 \mathrm{R} /$ receptor$/ 2 \mathrm{R}$ and conditionally protective ACE $2 \rightarrow$ ANG(1-7) $\rightarrow$ MasR receptor. The changes will contribute to the progression of inflammatory and thrombotic processes caused by local hyperactivity of $\mathrm{ANG}(1-8)$, which is not opposed by ANG(1-7). As an additional argument, clinical observations indicating that ACE inhibitors or BK receptor antagonists, which are commonly used to treat and prevent hypertension, are now associated with reduced disease severity and a trend toward low IL-6 levels with increased circulation of CD3/CD4/T cells (Xiao et al., 2020).

The third component of the regulation of the biochemical axes of ACE/ACE2 is bradykinin, a factor of the humoral kallikrein-kinin system with the involvement of the Hageman factor in this scheme. ACE, as a 
two-turn key, not only participates in the formation of prohypertensive ANG (1-8), but also, like kininase II, hydrolyzes bradykinin and limits its functions (Gomazkov and Komissarova, 1976). The physiological effects of kinins are mediated through the BK1R and $\mathrm{BK} 2 \mathrm{R}$ receptors in the vascular endothelium and smooth muscle cells. New data indicate that bradykinin, by interacting with these receptors, affects vascular permeability and cellular inflammation, inducing the expression of the cytokines IL-1, IL-2, IL-6, IL-8 and TNF- $\alpha$. It was also found that bradykinin stimulated the release of proinflammatory cytokines: neutrophils and chemotactic monocytes in alveolar cells (Koyama et al., 1998).

Previous work adds evidence about the role of the kallikrein-kinin system in the pathogenesis of pulmonary infection. In patients with acute respiratory distress in the bronchoalveolar lavage, the content of activated Hageman's factor (XIIa), prekallikrein, and high molecular weight kininogen is increased (McGuire et al., 1982). In the context of the analysis of the pathogenesis of COVID-19, it was proposed that angioedema during infection is associated with the activation of kinin receptors B1R and B2R. From a pharmacological evaluation, the blockage of $\mathrm{B} 2 \mathrm{R}$ receptors and inhibition of kallikrein activity may have a mitigating effect in the early period of COVID-19 disease and influence the development of acute respiratory syndrome (van de Veerdonk et al., 2020; Kreutz et al., 2020). Thus, the dysregulation caused by high kinin activity is present already in the early stages of a viral attack in the pathology of COVID-19. Blockade of the ACE2 enzyme by the coronavirus is becoming a precedent that stimulates the mechanism of the damaging action of bradykinin.

\section{STORM-2 AS A SECOND STAGE OF COVID-19 PATHOGENESIS}

Along with the primary, dramatic attack of the virus on the lung system, the most likely scenario for COVID-19 pathogenesis is damage to the endothelial cells of the lungs and other organs. Coronavirusinduced cytokine storm is considered a special clinical syndrome of a destructive, pro-inflammatory etiology with manifestations of hemodynamic instability, multiple organ dysfunction, neurological dissonance. Impaired interaction between endothelial and immune cells can play a major role both in the initial periods of the disease, especially in severe cases, and in the later stages.

The mechanism of the destructive role of the coronavirus seems to be significant against this background. It uses ACE2 as a target and levels the normal, functional balance of the ACE/ACE2 axis systems. ACE2 blockade and a change in the ratio in the angiotensin's-bradykinin ligament explain the damage to many organs.
Several positions can be distinguished from a summary of the data of clinical and experimental studies.

- A feature of COVID-19 pathology, in contrast to the previous forms of acute respiratory lesions, is a generalized disorder of hemovascular control. The vascular-lesion mechanism includes an imbalance with the prevalence of inflammatory, oxidative, vasoconstrictor reactions. These processes lead to changes in permeability, vascular tone, and the ratio of thrombin and plasmin blood systems. The complex of these reactions is the cause of damage to many organs.

- Disorder of the biochemical "axis" of the reninangiotensin and kallikrein-kinin systems turns out to be the main consideration for COVID-19 pathogenesis. An imbalance due to an increase in ACE activity, while leveling ACE2, leads to a limitation of the activity of bradykinin and an increase in ANG(1-8) as factors of proinflammation, thrombogenesis, and prohypertensive activity.

- The proposed concept, STORM-2, is based on the analysis of biochemical mechanisms. Its key component is endothelial dysfunction and "disorder" in the management of the blood-coagulation system, vascular tone, transcellular diffusion, and blood-pressure maintenance. Clinical analysis shows that patients with an acute course of COVID-19 develop vascular-endothelial dysfunction of the heart, brain, kidneys, endocrine, etc. systems, coagulopathy, and thrombosis with a clinical picture resembling catastrophic antiphospholipid syndrome. This process is primarily typical for the lungs, but it also refers to damage to many systems: the myocardium, kidneys, brain, gastrointestinal system, endocrine organs, etc. It is essential that complications are associated with the presence of other forms of pathology in the patient's history. Of particular importance is the direct and "delayed" impairment of brain functions, including the psychosocial aspect, which captures almost the entire modern world (Vremennye..., 2020). With the proposed term STORM-2, these clinical conclusions are confirmed by the illustration of the mechanisms of ACE/ACE2 dissonance, which implies that a selective therapy strategy can be used for COVID-19.

\section{CONCLUSIONS}

The purpose of this presentation was to systematize the information received in the last few months of the fight against the COVID-19 pandemic. A review of publications on COVID-19 and the author's experience in molecular pathophysiology made it possible to determine the main lines of presentation.

The virus SARS-CoV-2 triggers a cytokine storm and endothelial dysfunction. As a result, the organized physiological system turns into a mechanism of polyfunctional "disturbance" and disease development. Since the main target of the fixing of the pathogenic SARS-CoV-2 virus is the ACE2 enzyme, a representa- 
tive of the key control of the hematovascular system, consideration of its role in "raider reception" becomes the leading line of presentation.

The essence of the pathogenetic action of the coronavirus is a massive attack on ACE2 and the destruction of the physiological balance of the ACE/ACE2 complex. Since both enzymes are proteinases localized predominantly in endothelial cells, this tissue "bridgehead" turns out to be a place of pathological dissonance of the blood and blood vessel systems. The appearance of the SARS-CoV-2 virus with a selective ACE2 lesion fatally affects the picture of functional imbalance. The scheme associated with the pathogenesis of the SARS-CoV-2 coronavirus is a leveling of the ACE2 and an in increase the destructive activity of the ACE. The functional imbalance of ACE/ACE2 leads to an increase in the role of kinins and angiotensin II as factors of inflammation, cytokine initiation, increased thrombogenesis, and prohypertensive activity.

The STORM-2 concept is based on an analysis of materials that accentuate changes in the biochemical axis of angiotensin and kinin polypeptides, the main regulators of the blood state and vascular tone. A complex of pathochemical processes unfolds in the area of the endothelium, where multiple organ coagulopathy and disorders of macro- and microhemodynamics with multiple features of the lesion develop in the second phase of viral infection. This negative picture is typical not only for lung damage, in which the coronavirus impact is primary, but also for the heart, kidneys, endocrine organs, brain, and other body systems.

The STORM-2 concept implies the need to apply a special strategy for COVID-19 pharmacotherapy. The key component is normalization of the endothelial function and control of the blood-coagulation system, vascular tone, transcellular diffusion, and blood pressure with an effect on the corresponding pharmacological targets.

\section{ACKNOWLEDGMENTS}

The author is grateful to A.V. Rudik for support and technical support of the work.

\section{FUNDING}

This work was partially supported by the Russian Foundation for Basic Research (project no. 20-04-60285).

\section{COMPLIANCE WITH ETHICAL STANDARDS}

Conflict of interests. The author declares that he has no conflicts of interest.

Statement on the welfare of humans or animals. This article does not contain any studies involving humans or animals performed by the author.

BIOLOGY BULLETIN REVIEWS Vol. $11 \quad$ No. $6 \quad 2021$

\section{REFERENCES}

Barth, M., Bondoux, M., Luccarini, J.-M., et al., From bradykinin B2 receptor antagonists to orally active and selective bradykinin B1 receptor antagonists, J. Med. Chem., 2012, vol. 55, no. 6, pp. 2574-2584.

Donoghue, M., Hsieh, F., Baronas, E., et al., A novel angiotensin-converting enzyme-related carboxypeptidase (ACE2) converts angiotensin I to angiotensin 1-9, Circ. Res., 2000, vol. 87, pp. E1-E9.

https://doi.org/10.1161/01.res.87.5.e1

Dubilei, P.V., Urazaeva, Z.V., and Khamitov, Kh.S., Bar'ernaya funktsiya legkikh i obespechenie gomeostaza (Barrier Function of Lungs and Homeostasis), Kazan: Kazan. Gos. Univ., 1987.

Eliseeva, Yu.E. and Orekhovich, V.N., Isolation and study of the specificity of carboxycatepsin, Dokl. Akad. Nauk SSSR, 1963, vol. 153, no. 4, pp. 954-956.

Fang, C. and Schmaier, A.H., Novel antithrombotic mechanisms mediated by Mas receptor as result of balanced activities between the kallikrein/kinin and the reninangiotensin systems, Pharmacol. Res., 2020, vol. 160, p. 105096. https://doi.org/10.1016/j.phrs.2020.105096

Gheblawi, M., Wang, K., Viveiros, A., et al., Angiotensin converting enzyme 2: SARS-CoV-2 receptor and regulator of the renin-angiotensin system, Circ. Res., 2020, vol. 126 , no. 10 , pp. $1456-1474$. https://doi.org/10.1161/CIRCRESAHA.120.317015

Gomazkov, O.A., Molecular and physiological aspects of endothelial dysfunction. The role of endogenous chemical regulators, Usp. Fiziol. Nauk, 2000, vol. 31, no. 4, pp. $48-62$.

Gomazkov, O.A. and Komissarova, N.V., General mechanisms of biochemical regulation of kallikrein, coagulation and fibrinolytic blood systems, Usp. Sovrem Biol., 1976 , vol. 82, no. 6, pp. 356-370.

Gomazkov, O.A. and Trapeznikova, S.S., Biochemical and physiological characteristics of kininase and angiotensin-converting function of lungs, Usp. Sovrem Biol., 1978, vol. 86, no. 2 , pp. 259-268.

Hamming, I., Timens, W., Bulthuis, M., et al., Tissue distribution of ACE2 protein, the functional receptor for SARS coronavirus. A first step in understanding SARS pathogenesis, J. Pathol., 2004, vol. 203, pp. 631-637.

Kalinina, E.V., Posdnev, V.F., Komissarova, N.V., and Gomazkov, O.A., Effect of new peptide inhibitors on the ratio of angiotensin-I converting and kinin-degrading activities (ACE), Biochemistry (Moscow), 1997, vol. 62 , no. 3, pp. 247-250.

Koyama, S., Sato, E., Nomura, H., et al., Bradykinin stimulates type II alveolar cells to release neutrophil and monocyte chemotactic activity and inflammatory cytokines, Am. J. Pathol., 1998, vol. 153, no. 6, pp. 18851893.

Kreutz, R., Algharably, E., Ganten, D., et al., Renin-angiotensin-system (RAS) and COVID-19-on the prescription of RAS blockers, Dtsch. Med. Wochenschr., 2020 , vol. 145 , no. 10 , pp. 682-686.

Li, W., Moore, M., Vasilieva, N., et al., Angiotensin-converting enzyme 2 is a functional receptor for the SARS coronavirus, Nature, 2003, vol. 426, pp. 450-454. 
Liu, Y., Yang, Y., Zhang, C., et al., Clinical and biochemical indexes from 2019-nCoV infected patients linked to viral loads and lung injury, Sci. China Life Sci., 2020, vol. 63 , pp. 364-374. https://doi.org/10.1007/s11427-020-1643-8

Mahmudpour, M., Roozbeh, J., Keshavarz, M., et al., COVID-19 cytokine storm: the anger of inflammation, Cytokine, 2020, vol. 133, p. 155151.

https://doi.org/10.1016/j.cyto.2020.155151

Marchandot, B., Sattler, L., Jesel, L., et al., COVID-19 related coagulopathy: a distinct entity? J. Clin. Med., 2020, vol. 9 , no. 6 , p. 1651 .

https://doi.org/10.3390/jcm9061651

McGonagle, D., Sharif, K., O'Regan, A., et al., The role of cytokines including interleukin-6 in COVID-19 induced pneumonia and macrophage activation syndrome-like disease, Autoimmun. Rev., 2020, vol. 19, no. 6 , p. 102537.

https://doi.org/10.1016/j.autrev.2020.102537

McGuire, W.W., Spragg, R.G., Cohen, A.B., and Cochrane, C.G., Studies on the pathogenesis of the adult respiratory distress syndrome, J. Clin. Invest., 1982, vol. 69 , no. 3 , pp. 543-553. https://doi.org/10.1172/jci110480

Merad, M. and Martin, J.C., Pathological inflammation in patients with COVID-19: a key role for monocytes and macrophages, Nat. Rev. Immunol., 2020, vol. 20, no. 6, pp. $355-362$.

https://doi.org/10.1038/s41577-020-0331-4

Pearce, L., Davidson, S.M., and Yellon, D.M., The cytokine storm of COVID-19: a spotlight on prevention and protection, Expert Opin. Ther. Targets, 2020, vol. 24, no. 8 , pp. $723-730$. https://doi.org/10.1080/14728222.2020.1783243

Qadri, F. and Bader, M., Kinin B1 receptors as a therapeutic target for inflammation, Expert Opin. Ther. Targets., 2018, vol. 22, no. 1, pp. 31-44. https://doi.org/10.1080/14728222.2018.1409724

Rajendran, P., Rengarajan, T., Thangavel, J., et al., The vascular endothelium and human diseases, Int. J. Biol. Sci., 2013, vol. 9, no. 10, pp. 1057-1069. https://doi.org/10.7150/ijbs.7502

Ryan, J.W., Ryan, U.S., Schultz, D.R., et al., Subcellular localization of pulmonary angiotensin-converting enzyme (kininase II), Biochem. J., 1975, vol. 146, no. 2, pp. $497-499$. https://doi.org/10.1042/bj1460497

Sardu, C., Gambardella, J., Morelli, M.B., et al., Hypertension, thrombosis, kidney failure, and diabetes: is COVID-19 an endothelial disease? A comprehensive evaluation of clinical and basic evidence, J. Clin. Med., 2020, vol. 9, no. 5, p. 1417. https://doi.org/10.3390/jcm9051417

Shimabukuro-Vornhagen, A., Gödel, P., Subklewe, M., et al., Cytokine release syndrome, J. Immunnother. Cancer, 2018, vol. 6, p. 56. https://doi.org/10.1186/s40425-018-0343-9

Skeggs, I., Kahn, J., and Shumway, N.P., The preparation and function of the hypertensin converting enzyme, $J$. Exp. Med., 1956, vol. 103, no. 3, pp. 295-299. https://doi.org/10.1084/jem.103.3.295

Souza Santos, R.A., Oliveira Sampaio, W., Alzamora, A.C., et al., The ACE2/angiotensin-(1-7)/MAS axis of the renin-angiotensin system: focus on angiotensin-(1-7), Physiol. Rev., 2018, vol. 98, no. 1, pp. 505-553.

Tai, W., He, L., Zhang, X., et al., Characterization of the receptor-binding domain (RBD) of 2019 novel coronavirus: implication for development of RBD protein as a viral attachment inhibitor and vaccine, Cell Mol. Immunol., 2020, vol. 17, p. 613. https://doi.org/10.1038/s41423-020-0400-4

Tang, N., Li, D., Wang, X., and Sun, Z., Abnormal coagulation parameters are associated with poor prognosis in patients with novel coronavirus pneumonia, J. Thromb. Haemostasis, 2020, vol. 18, pp. 844-847.

Tipnis, S., Hooper, N.M., Hyde, R., et al., A human homolog of angiotensin-converting enzyme. Cloning and functional expression as a captopril-insensitive carboxypeptidase, J. Biol. Chem., 2000, vol. 275, p. 33238. https://doi.org/10.1074/jbc.M002615200

Vane, J.R., The release and fate of vasoactive hormones in the circulation, Br. J. Pharmacol., 1969, vol. 35, no. 2, pp. 209-242. https://doi.org/10.1111/j.1476-5381.1969.tb07982.x

Vane, J.R., My life and times with enzymes and mediators, Med. Sci. Monit., 2001, vol. 7, pp. 790-800.

van de Veerdonk, F.L., Netea, M.G., van Deuren, M., et al., Kallikrein-kinin blockade in patients with COVID-19 to prevent acute respiratory distress syndrome, eLife, 2020, vol. 27, no. 9, p. e57555. https://doi.org/10.7554/eLife.57555

Verdecchia, P., Cavallini, C., Spanevello, A., et al., The pivotal link between ACE2 deficiency and SARS-CoV-2 infection, Eur. J. Int. Med., 2020, vol. 76, pp. 14-20. https://doi.org/10.1016/j.ejim.2020.04.037

Victorino, G.P., Newton, C.R., and Curran, B., Effect of angiotensin II on microvascular permeability, J. Surg. Res., 2002, vol. 104, pp. 77-81. https://doi.org/10.1006/jsre.2002.6412

Vremennye metodicheskie rekomendatsii: profilaktika, diagnostika i lechenie novoi koronavirusnoi infektsii COVID-19. Versiya 8 ot 3 sentyabrya 2020 (Interim Guidelines for Prevention, Diagnosis, and Treatment of Novel COVID19 Coronavirus Infection, Version 8 of September 3, 2020), Moscow, 2020.

Warner, F.J., Smith, A.I., Hooper, N.M., and Turner, A.J., What's new in the renin-angiotensin system? Angiotensin-converting enzyme-2: a molecular and cellular perspective, Cell. Mol. Life Sci., 2004, vol. 61, no. 21, pp. 2704-2713.

Williams, B., Baker, A.Q., Gallacher, B., et al., Angiotensin II increases vascular permeability factor gene expression by human vascular smooth muscle cells, Hypertension, 1995, vol. 25, no. 5, pp. 913-917.

Xiao, G., Zhang, J., He, X., et al., Renin-angiotensin system inhibitors improve the clinical outcomes of COVID-19 patients with hypertension, Emerging $\mathrm{Mi}$ crobes Infect., 2020, vol. 9, no. 1, pp. 757-760.

Ye, Q., Wang, B., and Mao, J., The pathogenesis and treatment of the 'cytokine storm' in COVID-19, J. Infect., 2020, vol. 80, no. 6, pp. 607-613.

Zou, X., Chen, K., Zou, J., et al., The single-cell RNA-seq data analysis on the receptor ACE2 expression reveals the potential risk of different human organs vulnerable to 2019-nCoV infection, Front Med., 2020, vol. 14, no. 2, pp. $185-192$. https://doi.org/10.1007/s11684-020-0754-0 